\title{
HER2 in gastric cancer: a biomarker with clinical impact, but not without translational challenges
}

\author{
Florian Lordick
}

$\mathrm{T}$ wenty-five years ago the amplification of the HER2 gene and its protein overexpression was first described in gastric cancer [1]. Twenty years later a group of Spanish investigators first experienced the activity of trastuzumab in patients with HER2 overexpressing gastric cancer [2]. Meanwhile, a randomised multi-national trial (ToGA) made things even clearer and brought trastuzumab to regulatory approval and marketing authorisation in Europe and other parts of the world. ToGA could show that the addition of trastuzumab to standard chemotherapy can prolong the survival of patients with incurable HER2 overexpressing gastric cancer [3]. The benefit is somewhere between 2.7 months (primary endpoint: all included patients) and 5.6 months (subgroup of patients with HER2 gene amplification and score $3+$ protein expression). Things look simple at first glance: Trastuzumab, a monoclonal antibody directed against the HER2 receptor protein, is effective in HER2-positive advanced gastric cancer and should be used in this disease in combination with standard platinum-5FU chemotherapy. But oncology would not be oncology if things remained so simple at a more in-depth analysis. Our current challenge with HER2-positive gastric cancer is to make the true nature of every individual disease visible and to translate our findings to the best available treatment recommendation. Our translator in this critical venture is our highly respected partner, the molecular pathologist, whomore than ever-is an integral part of the multidisciplinary treatment team.

Pathologists and clinicians know that-for the best of our patients and for pharmaco-economic reasons-"drugable targets" like the HER2 receptor need to be explored with robust and reliable methods. The tests we use should have a strong predictive value, which means a positive test result goes along with a high probability that the drug works

F. Lordick (ه)

Department of Hematology and Oncology

Klinikum Braunschweig

Celler Str. 38

38114 Braunschweig, Germany

e-mail: medklinik3@klinikum-braunschweig.de while a negative test result excludes meaningful activity of the corresponding drug.

There is no doubt that HER2 is a "drugable target" in gastric cancer, but is it also a robust predictive biomarker?

The paper of Gomez-Martin et al. delineates the technical and biological challenges we encounter when detecting and scoring HER2 as a biomarker in gastric cancer [4]. It would go far beyond the scope of this editorial to repeat all the details. But it is strongly recommended to read the paper. If someone thinks that the detection of a biomarker established in another disease like HER2 in breast cancer is a home run in gastric cancer, he or she will learn that this is not the case. But the good news is that the challenge is manageable if some strict rules are respected and a relatively robust predictive biomarker is available if the multidisciplinary team encompassing the surgeon, the endoscopist, the pathologist and the oncologist adheres to the given recommendations.

Gastric cancer is a changeable disease with many faces, presenting as tumour of the proximal, middle or distal parts of the stomach, with an intestinal or a diffuse growth pattern under the light microscope, all these conditions being associated with a different cancerogenesis and with different molecular features. Above all, gastric cancer often presents heterogenously in itself. Intra-tumoral heterogeneity is a real challenge for the establishment of a biomarker. The question remains: if in one part of the tumour the biomarker is found to be overexpressed, is this finding representative for the whole tumour, does it drive the natural course of the disease and does it signify that this particular cancer can be therapeutically addressed via this target? The current guidelines recommend that at least $10 \%$ of a resection specimen or at least 5 adjacent cells in endoscopic tumour biopsies should stain positive for HER2. In contrast to breast cancer, the apical membranes of the glandular cells do not need to stain positive for HER2; baso-lateral or lateral membrane staining alone is accepted. In the SEOM and SEAP consensus paper, detailed and pragmatic information is given on how to do the pre-analytic handling of tumour probes and how to assess HER2 protein expression by immuno-histochemistry (IHC) and HER2 gene amplification using fluorescence in 
situ hybridisation (FISH) or newer bright-field in situ hybridisation techniques $[4,5]$.

While the technological issues of HER2 detection seem to be solved as adequately as possible, many of the biological questions remain unanswered. It remains unclear why so many (almost 20\%) tumours with HER2 gene amplification (HER2:CEP17 ratio $\geq 2$ ) do not overexpress HER2 protein on their cell surface. Do we need to modify the amplification thresholds? Are there more posttranscriptional processes involved than we know yet? Other questions are equally important, like: What is the concordance of HER2 expression between primary tumours and metastases? Why does the expression of the oncogene HER2 not exhibit a clear prognostic value according to currently published studies? Is the HER2 expression level a more or less stable tumour characteristic or is there a shift over longer periods of time? What are the mechanisms of escape and resistance to anti-HER2 directed treatment? Does the efficacy of antiHER2 treatment observed in metastatic disease hold true for the adjuvant or neoadjuvant setting? These questions and others need to be and are being addressed in current and future clinical trials.

Beside the individual medical need of establishing HER2 as a robust predictive biomarker, there is also a socio-economic aspect that should not be neglected. With every patient treated with trastuzumab without a clear biologic justification, we make a bad investment of the capital of the social solidarity community into something that will be without benefit. Even in the richest societies we have limited resources for covering all medical needs.

In the United Kingdom the society seems to accept a more rationalistic and economy-driven decision making on medical treatment decisions and resource allocation. The UK National Institute for Health and Clinical Excellence (NICE) has issued that only patients with IHC score $3+$ HER2 overexpressing tumours will get the chance to be treated with trastuzumab on the expenses of the national health system. The justification is derived from the ToGA trial, which has shown a median gain in lifetime for IHC score $3+$ patients of 5.6 months, amounting to $£ 45,000-50,000$ to be spent per quality-adjusted life-year gained. The committee agreed that this amount of money was acceptable for an end-of-life patient population. In contrast, IHC $2+/$ FISH+ patients have a benefit of only 1.8 months according to ToGA. Therefore, when IHC Her2 2+/ FISH-positive patients are included in the treatment recommendation (like the marketing authorisation for trastuzumab allows), the median gain in lifetime shrinks to only 4.2 months, amounting to $£ 63,100-71,500 /$ quality-adjusted life-year gained, being an unacceptable amount of money according to NICE [6]. Of note, NICE thereby limits the indication to a subgroup of patients being allowed to receive the drug according to the European Medical Agency's marketing authorisation.

Many of us do not feel comfortable with economically driven regulations on this basis, but this process is certainly very interesting. For the first time to my knowledge, a health system bases a reimbursement embargo not on a whole drug or indication, as we have seen with bevacizumab for use in breast cancer in the USA. In the case of HER2-positive gastric cancer the expression level of a biomarker forms the basis for such a decision, putting the accuracy of the diagnostic process at the centre of interest.

The testing algorithm for HER2 in advanced gastric cancer, as published in this issue of Clinical and Translational Oncology [4, 5], represents the best available recommendation at present. The teams and diagnostic institutions involved in the treatment of gastric cancer should absolutely adhere to it. But work should certainly go on. The task is not to count as many "HER2 positives" as possible (as some drug manufacturers may wish) but to classify as adequately as possible. False negative findings lead to missed therapeutic chances for the individual patient, thereby limiting his potential life-expectancy, while false positive findings lead to unnecessary risks for toxicity, to prolonged infusion times and to a considerable waste of money. Hopefully, in future test algorithms for gastric cancer we will find even more accurate information about the prediction of response and resistance to anti-HER2 targeting agents. Test algorithms for other targeted drugs may follow.

The Spanish Societies of Medical Oncology and Pathology are to be congratulated for undertaking this very important multidisciplinary initiative and outlining all necessary details of HER 2 testing in a most valuable publication. All oncologists and pathologists are encouraged to take part in the SEAP quality assurance programme for HER2 testing in gastric cancer.

\section{References}

1. Yamamoto T, Ikawa S, Akaiyama T et al (1986) Similarity of protein encoded by the human cerb-B-2 gene to epidermal growth factor receptor. Nature 319:230-234

2. Cortes-Funes H, Rivera F, Ales I et al (2007) Phase II trial of trastuzumab and cisplatin in patients (pts) with advanced gastric cancer (AGC) with Her2/neu overexpression/amplification. J Clin Oncol 2007 (ASCO Annual Meeting Pro- ceedings) Part I. 26[18S, June 20 Suppl]:abstract 4613

3. Bang YJ, Van Cutsem E, Feyereislova A et al (2010) Trastuzumab in combination with chemotherapy versus chemotherapy alone for the treatment of HER2 positive advanced gastric or gastro-oesophageal junction cancer (ToGA): a phase 3, open-label, randomized controlled trial. Lancet 376:687-697

4. Gomez-Martin C, Concha A, Corominas JM et al (2011) Consensus of the Spanish Society of
Medical Oncology (SEOM) and Spanish Society of Pathology (SEAP) for HER2 testing in gastric carcinoma. Clin Transl Oncol 13:636-651

5. Ruschoff J, Dietel M, Baretton G et al (2010) HER2 diagnostics in gastric cance: guideline validation and development of standardized immunohistochemical testing. Virchows Arch 457:299-307

6. National Health Service (2010) NICE technology appraisal guidance 208: trastuzumab for the treatment of HER2-positive metastatic gastric cancer. November 